\title{
Antiepileptic drugs for children: Availability, suitability and acceptability
}

\author{
UL Somasiri $^{1}$, S Thillainathan ${ }^{1}$, R Fernandopulle ${ }^{2}$, S Sri Ranganathan ${ }^{2}$
}

Sri Lanka Journal of Child Health, 2012; 41(1): 38-39

(Key words: Antiepileptic drugs; children; availability; suitability; acceptability)

\section{Background}

Effective treatment of epilepsy requires consistent plasma steady state concentration of the antiepileptic drug (AED). This calls for rational selection of the AED and an appropriate dosing regimen. A recent survey of medicines issued to the state sector indicated a shortfall in age-appropriate formulations of paediatric AEDs ${ }^{1}$. Unavailability of the suitable paediatric formulation is overcome by one of two ways; extemporaneous preparation (or compounding) of liquid formulations or manipulation of adult dosage-form. Such manipulation could reduce the bioavailability as many AEDs are sparingly soluble in aqueous solutions and sensitive to effects that alter solubility or dissolution. Further, compliance may be affected as the palatability of manipulated dosage-forms has not been studied.

This study describes the availability of AEDs in suitable paediatric formulations at the Lady Ridgeway Hospital (LRH) for Children, methods of manipulation adapted by parents and palatability, acceptability and accuracy of dose of such manipulated dosage forms.

\section{Methods}

A prospective cross sectional study was conducted at the OPD pharmacy of the LRH. Study population comprised children aged 12 and below with epilepsy who regularly attend the clinic and collect their AEDs from the OPD pharmacy. A consecutive sample of 109 children was recruited in one week in the latter part of 2008. Children attending the clinic for the first time were excluded. The investigators visited the pharmacy, identified the children, explained the study to the parents, gave an information sheet and obtained consent.

Data was collected from parents using a pre-tested,

${ }^{1}$ Medical students, Faculty of Medicine, University of Colombo, Sri Lanka, ${ }^{2}$ Department of Pharmacology, Faculty of Medicine, University of Colombo, Sri Lanka

(Received on 12 February 2012; Accepted on 23 March 2012) structured, interviewer administered questionnaire, analysed and presented using simple descriptive statistics. Study protocol was approved by both Ethics Review Committees of the Faculty of Medicine, University of Colombo and LRH.

\section{Results}

The mean age of study sample was 6.3 years $(\mathrm{SD}=$ 3.37 ), 15 children in $0-2$ year, 38 in 2-6 year and 56 in $6-12$ year age groups. They were on eight different AEDs with the majority $(63 \%)$ on monotherapy. Sodium valproate $(57 \%)$ was the most frequently prescribed AED followed by carbamazepine $(16 \%)$. None of the prescribed AEDs were available in suitable paediatric formulations. Except a 2 year old for whom parents were buying sodium valproate syrup from private pharmacies, the others, irrespective of their ages, received adult strength AED tablets. Parents are instructed by pharmacists to segment the unscored adult tablet. Of the 53 children between $0-6$ years, parents segmented the adult tablet: in $45 \%$ into half, in $7 \%$ into quarter, in $6 \%$ into three-quarter and in 3\% into one-eighth.

Parents used diverse methods to encourage children swallow the whole or segmented tablet. Majority in 2-6 (60.5\%) and $6-12$ year $(78.5 \%)$ age groups managed to swallow the tablet whole/part. A small minority $(13.3 \%)$ of children in $0-2$ year age group chewed the AED with the rest receiving it crushed and mixed with a variety of vehicles including water $(54.5 \%)$, breast milk (17\%), honey, sugar, co-prescribed syrup and food.

On assessing the palatability of these medicines in the 5-12 year age group using a Likert scale (1-very tasty, 5 - very bitter), majority graded valproate $(65 \%)$ and carbamazepine $(64 \%)$ as of average taste. Approximately one-fifth of parents always encountered problems in administering valproate with half finding it hard to crush; when broken, the remaining half for the next dose often disappears (melts) and children refusing it claiming it was very bitter. This resulted in out of pocket purchasing till the next clinic date. 


\section{Interpretation}

Despite the regulatory authorisation of paediatric formulations of AEDs and inclusion of liquid formulations of carbamazepine and sodium valproate in the National List of Essential Medicines, young children received manipulated adult dosage forms. Though the study reports one week data, the pharmacists at LRH reported that they rarely had liquid formulations of AEDs.

Tablet splitting of AEDs by parents on the instructions of pharmacist to overcome stock-outs seems to be a common practice at LRH. The practice of segmenting tablets assumes there is uniform distribution of the active drug within the tablet and the ability to segment the tablet accurately. Rosenberg et $\mathrm{al}^{2}$ determined the level of weight uniformity of segments from tablets cut into halves by pharmacists. 560 split tablet halves were collected and their weights, determined using criteria from the USP. They concluded that tablet splitting resulted in an unacceptably high incidence of weight variation. It is also accepted that the act of crushing tablets which are not designed to be administered in this way and using untested vehicles, may alter the pharmacokinetics of the medication $^{3}$. This could be significant for drugs with a narrow therapeutic range such as AEDs. We recommend that "Child Friendly" formulations and strengths be made available for medicines such as AEDs which need consistent drug concentrations in the plasma for effective treatment. Methods used by parents to make their children swallow the AEDs are shown in Table 1.

Table 1: Methods used by parents to make their children swallow the AEDs

\begin{tabular}{|l|c|c|c|c|}
\hline \multicolumn{1}{|c|}{ Methods } & $\mathbf{0 - 2 y r s}$ & $\mathbf{2 - 6 y r s}$ & $\mathbf{6 - 1 2 y r s}$ & Total \\
\hline Swallows whole/part of the tablet with water & 0 & 23 & 44 & 67 \\
\hline $\begin{array}{l}\text { Swallows broken/crushed tablet mixed with various } \\
\text { vehicles }\end{array}$ & 13 & 14 & 8 & 35 \\
\hline Swallows chewed tablet (whole/part) with water & 2 & 1 & 4 & 7 \\
\hline Total & 15 & 38 & 56 & 109 \\
\hline
\end{tabular}

\section{Acknowledgements}

We acknowledge the Director, LRH for granting permission to collect data and pharmacists at the LRH/OPD pharmacy for assisting in the data collection. We acknowledge the Elective Committee, Faculty of Medicine, University of Colombo, as this research was done through the elective programme offered to undergraduates

\section{References}

1. Sri Ranganathan S. Fernandopulle R, Beneragama B V H, Weerasinghe $\mathrm{MC}$, Weeraratne ED. An Analysis of the pharmaceuticals issued by the Medical Supplies Division (MSD) over a period of five years; 2002-2006 - Sri Lanka; 2009

2. Rosenberg JM et al. Weight variability of pharmacist dispensed split tablets. Journal of American Pharmacists Association, 2002; 42(2): 200-5. http://dx.doi.org/10.1331/10865800276350849 $\underline{8}$

3. Notterman DA. Effect of dose formulations on isoniazid absorption in 2 young children. Pediatrics, 1986; 77(6): 850-2. 